\title{
Application of the optimized Baxter model to the hard-core attractive Yukawa system
}

\author{
Peter Prinsen ${ }^{\text {a) }}$ \\ Complex Fluids Theory, Faculty of Applied Sciences, Delft University of Technology, Julianalaan 67, \\ 2628 BC Delft, The Netherlands \\ Josep C. Pàmies \\ FOM Institute for Atomic and Molecular Physics, Kruislaan 407, 1098 SJ Amsterdam, The Netherlands \\ Theo Odijk \\ Complex Fluids Theory, Faculty of Applied Sciences, Delft University of Technology, Julianalaan 67, \\ 2628 BC Delft, The Netherlands \\ Daan Frenkel \\ FOM Institute for Atomic and Molecular Physics, Kruislaan 407, 1098 SJ Amsterdam, The Netherlands
}

(Received 27 March 2006; accepted 17 October 2006; published online 16 November 2006)

We perform Monte Carlo simulations on the hard-core attractive Yukawa system to test the optimized Baxter model that was introduced by Prinsen and Odijk [J. Chem. Phys. 121, 6525 (2004)] to study a fluid phase of spherical particles interacting through a short-range pair potential. We compare the chemical potentials and pressures from the simulations with analytical predictions from the optimized Baxter model. We show that the model is accurate to within $10 \%$ over a range of volume fractions from 0.1 to 0.4 , interaction strengths up to three times the thermal energy, and interaction ranges from $6 \%$ to $20 \%$ of the particle diameter, and performs even better in most cases. We furthermore establish the consistency of the model by showing that the thermodynamic properties of the Yukawa fluid computed via simulations may be understood on the basis of one similarity variable, the stickiness parameter defined within the optimized Baxter model. Finally, we show that the optimized Baxter model works significantly better than an often used, naive method determining the stickiness parameter by equating the respective second virial coefficients based on the attractive Yukawa and Baxter potentials. () 2006 American Institute of Physics. [DOI: $10.1063 / 1.2390699$ ]

\section{INTRODUCTION}

In a recent paper $^{1}$ two of us devised a method to approximate systematically a system of spherical hard particles that interact through a short-range pair potential by a system of particles interacting via an effective Baxter potential. ${ }^{2}$ The latter consists of a hard-core repulsion and a sticky attraction at the surface of the particles which is computed by a variational principle for the free energy (hence the name "optimized Baxter model"1). The original short-range potential was a sum of attractive and repulsive contributions (i.e., a square well plus a Debye-Hückel interaction ${ }^{1}$ ), but the variational method also applies to a purely attractive interaction provided its range is sufficiently smaller than the particle diameter. The advantage of approximating the original interaction by the Baxter potential is that the fluid phase of the Baxter model has been studied extensively, both theoretically ${ }^{2-9}$ and in computer simulations. ${ }^{10-13}$ This means that, once the correspondence between the two systems has been established, all the analytical results of the Baxter model can be fruitfully used for the original system.

In the optimized Baxter model (OBM) ${ }^{1}$ the free energy

\footnotetext{
${ }^{a)}$ Author to whom correspondence should be addressed. Electronic mail: p.prinsen@tnw.tudelft.nl
}

of the actual system is functionally expanded in terms of the Mayer function, where the reference state is a suspension of hard spheres interacting via an effective sticky potential. The stickiness parameter associated with the latter is determined by setting the first-order term in this expansion equal to zero. This constitutes a variational principle because the secondorder term turns out to be either positive or negative definite. ${ }^{1}$ Nevertheless, the exact nature of the expansion is difficult to assess analytically. For instance, there may be mathematical problems arising from the limiting procedure in which the range of the effective adhesion goes to zero as its magnitude becomes infinitely large. ${ }^{1}$ Thus, a computational test of the OBM is important.

The model system we consider consists of hard-sphere particles with an attractive Yukawa interaction,

$$
\frac{U_{Y}(x)}{k_{B} T}= \begin{cases}\infty, & 0 \leqslant x<2 \\ -\beta \frac{e^{-\kappa(x / 2-1)}}{x / 2}, & x \geqslant 2 .\end{cases}
$$

Here, $x \equiv r / a$ is the dimensionless distance between the centers of mass of two spheres, $a$ is their radius, $k_{B}$ is Boltzmann's constant, $T$ is the temperature, $\beta$ is the dimensionless well depth, and $a / \kappa$ is a measure of the range of the attractive tail (if we wish to set the actual well depth $\equiv$ unity, $\beta$ 
may be viewed as identical with $\left.1 / k_{B} T\right)$. Note that we scale distances by $a$ here although often distances are scaled by the diameter $2 a$. $^{14,15}$

The liquid-solid coexistence of this system has been studied before at various values of $\kappa \cdot{ }^{14,15}$ These papers do not report the chemical potentials and pressures at coexistence, however, which we need to test the validity of the OBM. We therefore perform new simulations to determine the volume fraction, chemical potential, and pressure at various points along the phase boundaries. Moreover, we also determine the chemical potential and pressure within the fluid region of the phase diagram so as to gauge the accuracy of the OBM at lower concentrations.

We start by reviewing equations relevant to the $\mathrm{OBM}$ as applied to the Yukawa potential [Eq. (1)] in the next section. In Sec. III we describe the numerical simulations which, in Sec. IV, are compared with the theoretical predictions.

\section{THEORY}

Here we give a short summary of the theory developed in Ref. 1. The relevant equations needed to determine the effective adhesion parameter $\tau$ and some of the thermodynamic properties of the system are presented here. For details of the derivation we refer to Ref. 1 and references mentioned there.

We consider a system of spherical particles of radius $a$. The interaction $U$ between the particles is pairwise additive and consists of a hard-sphere repulsion plus a short-range interaction $U_{1}$ that is either purely attractive or consists of a combination of attractive and repulsive interactions (range $\ll a)$. In the latter case, the attraction has to be strong enough to compensate for the repulsion-we will come back to this issue later. For convenience, all distances are scaled by the radius $a$ of the particles so we have

$$
U(x)= \begin{cases}\infty, & 0 \leqslant x<2 \\ U_{1}(x), & x \geqslant 2\end{cases}
$$

We wish to replace this system by a suspension of adhesive hard spheres with the same radius which is our reference state. The interaction of the latter is given by the adhesive hard sphere (AHS) potential of Baxter, ${ }^{2}$

$$
\frac{U_{\mathrm{AHS}}(x)}{k_{B} T}= \begin{cases}\infty, & 0 \leqslant x<2 \\ \ln \frac{12 \tau \omega}{2+\omega}, & 2 \leqslant x \leqslant 2+\omega \\ 0, & x>2+\omega .\end{cases}
$$

Here, $\tau$ is the stickiness parameter whose magnitude we wish to determine and which signifies the strength of the effective adhesion. The limit $\omega \downarrow 0$ has to be taken after formal integrations. The reason for approximating the original system by the AHS system is that the latter has been conveniently solved in the Percus-Yevick approximation. ${ }^{2}$ This means that once the correspondence between the two systems has been established by appropriately choosing $\tau$, other properties such as the chemical potential, the pressure, and the compressibility of the system can easily be computed analytically from the solution of the AHS system.
We next describe how to choose the stickiness parameter $\tau$. In the limit of vanishing densities, this is done by equating second virial coefficients since we must equate the respective free energies of the two systems.

$$
\begin{aligned}
B_{2} & =2 \pi a^{3} \int_{0}^{\infty} d x x^{2}\left(1-e^{-U(x) / k_{B} T}\right) \\
& \equiv B_{2}^{\mathrm{AHS}}=B_{2}^{\mathrm{HS}}\left(1-\frac{1}{4 \tau_{0}}\right) .
\end{aligned}
$$

This amounts to choosing

$$
\tau_{0}=\frac{2}{3 \int_{2}^{\infty} d x x^{2}\left(e^{-U_{1}(x) / k_{B} T}-1\right)} .
$$

Here, $B_{2}^{\mathrm{HS}}=16 \pi a^{3} / 3$ is the second virial coefficient of a solution of hard spheres. At finite densities this procedure necessarily breaks down, however, because the higher virials come into play. The stickiness parameter $\tau$, which depends on the density, has to be obtained by identifying the free energy of the actual system with that of the reference state as well as possible. In the functional expansion of the excess free energy in terms of the Mayer function, ${ }^{16}$ we then demand that the first-order correction vanishes. This leads to the condition ${ }^{1}$

$$
\int_{0}^{\infty} d x x^{2}\left(e^{-U(x) / k_{B} T}-1\right) \widetilde{g}(x)=\frac{2 \lambda}{3},
$$

where $\widetilde{g}(x)$ is the regular part of the pair correlation function $g(x)$ of the reference AHS system (which also has a singular term $\left.^{1,2}\right)$ and

$$
\lambda=\frac{6(\eta+(1-\eta) \tau)}{\eta(1-\eta)}\left(1-\sqrt{1-\frac{\eta(2+\eta)}{6(\eta+(1-\eta) \tau)^{2}}}\right),
$$

with $\eta$ the volume fraction of particles. For $x<2, \widetilde{g}(x)$ equals zero owing to the hard-core repulsion, whereas $\widetilde{g}(x)$ tends to unity for large $x$. Since the interaction $U(x)$ is of short range, we approximate $\widetilde{g}(x)$ in the interval $2 \leqslant x \leqslant 4$ by the first two terms of its Taylor expansion ${ }^{1}$

$$
\widetilde{g}(x) \simeq \begin{cases}0, & x<2 \\ G(1+H(x-2)), & 2 \leqslant x \leqslant 4 \\ 1, & x>4 .\end{cases}
$$

Here, we define the functions

$$
G=G(\eta, \tau)=\lambda \tau
$$

and

$$
\begin{aligned}
H=H(\eta, \tau)= & \frac{\eta}{2 \tau(1-\eta)}\left(\frac{\eta(1-\eta)}{12} \lambda^{2}-\frac{1+11 \eta}{12} \lambda\right. \\
& \left.+\frac{1+5 \eta}{1-\eta}-\frac{9(1+\eta)}{2(1-\eta)^{2}} \frac{1}{\lambda}\right) .
\end{aligned}
$$

At a given volume fraction $\eta, \tau$ can then be determined iteratively from Eqs. (6)-(10). An iterative scheme, which converges fast, consists of choosing a starting value of $\tau$, determining $\lambda$ from Eq. (7), then $\tau$ from 


$$
\tau=\frac{2 \lambda-3 \int_{4}^{\infty} d x x^{2}\left(e^{-U(x) / k_{B} T}-1\right)}{3 \lambda \int_{2}^{4} d x x^{2}(1+H(x-2))\left(e^{-U(x) / k_{B} T}-1\right)},
$$

$\lambda$ again from Eq. (7) and so on until convergence to the required accuracy is achieved.

There are two cases in which the above method does not yield meaningful results. The first occurs when the shortrange interaction has both attractive and repulsive components in the event that the repulsion is too strong in comparison with the attraction. The total interaction is then effectively repulsive in nature, so it is clear that a suspension of particles interacting in such a way cannot be approximated by an AHS system. In this case, the iteration scheme described above leads to a $\tau$ which keeps on increasing and does not converge. If $\tau_{0}$ is negative in the limit of vanishing density [Eq. (5)] implying a net repulsion, it is advisable not to compute $\tau$ in that case, even though $\tau$ could attain positive values at higher densities. Secondly, the attraction may be too strong. There exists a critical value of the stickiness parameter $\tau_{c}$ below which there is a range of densities for which there is no real solution of $\lambda$

$$
\tau_{c}=\frac{2-\sqrt{2}}{6} \text {. }
$$

This means that if the attraction is strong enough (i.e., when $\tau$ is too small), there will not be a positive real solution to Eq. (11). In this case the iteration scheme would produce complex values of $\tau$.

We now state several thermodynamic properties resulting from the solution of the Baxter model which we will need further on. To compute the pressure $P$ and the chemical potential $\mu$ we use the expressions derived via the compressibility route, ${ }^{2,4}$

$$
\frac{P v_{0}}{k_{B} T}=\frac{\eta\left(1+\eta+\eta^{2}\right)}{(1-\eta)^{3}}-\frac{\eta^{2}(1+\eta / 2)}{(1-\eta)^{2}} \lambda+\frac{\eta^{3}}{36} \lambda^{3}
$$

and

$$
\frac{\mu-\mu_{0}}{k_{B} T}=\ln \frac{\eta}{1-\eta}+\frac{3 \eta(4-\eta)}{2(1-\eta)^{2}}+\frac{P v_{0}}{k_{B} T}+J .
$$

Here $v_{0}=4 \pi a^{3} / 3$ is the volume of a particle,

$$
\begin{aligned}
J= & \frac{3}{2} \eta^{2} \lambda^{2}-\frac{3 \eta(1+4 \eta)}{(1-\eta)} \lambda+\frac{6 \eta(2+\eta)}{(1-\eta)^{2}}-\frac{18 \eta}{1-\eta} \tau \\
& -\frac{6\left(\tau-\tau_{c}\right)^{2}}{\tau_{c}\left(1-6 \tau_{c}\right)} \ln \left|\frac{\lambda(1-\eta)-\tau_{c}^{-1}}{\tau^{-1}-\tau_{c}^{-1}}\right| \\
& +\frac{6 \tau_{c}\left(18 \tau \tau_{c}-1\right)^{2}}{1-6 \tau_{c}} \ln \left|\frac{\lambda(1-\eta)-18 \tau_{c}}{\tau^{-1}-18 \tau_{c}}\right|
\end{aligned}
$$

is the contribution to the chemical potential that vanishes in the hard-sphere limit $(\tau \rightarrow \infty)$ and the chemical potential of the reference state (in the context of the Baxter model) is given by

$$
\frac{\mu_{0}}{k_{B} T}=\ln \frac{1}{v_{0}}\left(\frac{h^{2}}{2 \pi m k_{B} T}\right)^{3 / 2},
$$

\section{SIMULATIONS}

We perform Monte Carlo simulations at constant volume $V$ and temperature $T$ on a system of $N=256$ hard spheres with a short-range Yukawa attraction, so we have [compare with Eq. (1)]

$$
\frac{U_{1}(x)}{k_{B} T}=-\beta \frac{e^{-\kappa(x / 2-1)}}{x / 2} \text {. }
$$

We introduce a cutoff at $x=4$, so that $U(x)=0$ for $x>4$. We determine the Helmholtz free energy per particle $f_{N}$ at a chosen set of parameters of $\beta, \kappa$, and $\eta$ by thermodynamic integration at constant $\kappa$ and $\eta$, starting from the known free energy per particle of the hard-sphere system $(\beta=0)$, which is defined at the same volume fraction (see, e.g., Ref. 17),

$$
\frac{f_{N}(\eta, \beta)-f_{N}(\eta, 0)}{k_{B} T}=\int_{0}^{\beta} d \beta^{\prime} \frac{1}{\beta^{\prime}}\left\langle\frac{U_{1}}{k_{B} T}\right\rangle_{N} .
$$

Here $\left\langle U_{1}\right\rangle_{N}$ is the average energy per particle where the average is computed in the state with $\beta=\beta^{\prime}$. From this we determine the equation of state $z_{N}(\eta, \beta)$

$$
\begin{aligned}
z_{N}(\eta, \beta)-z_{N}(\eta, 0) & =\frac{P_{N}(\eta, \beta)}{\rho k_{B} T}-\frac{P_{N}(\eta, 0)}{\rho k_{B} T} \\
& =\eta \frac{\partial}{\partial \eta}\left[\frac{f_{N}(\eta, \beta)-f_{N}(\eta, 0)}{k_{B} T}\right],
\end{aligned}
$$

where the particle density $\rho$ is related to the volume fraction by $\rho v_{0}=\eta$, and the chemical potential $\mu_{N}(\eta, \beta)$ is given by

$$
\frac{\mu_{N}(\eta, \beta)}{k_{B} T}-\frac{\mu_{N}(\eta, 0)}{k_{B} T} \simeq \frac{\partial}{\partial \eta} \eta\left[\frac{f_{N}(\eta, \beta)-f_{N}(\eta, 0)}{k_{B} T}\right] .
$$

The expression for the pressure is exact for a system consisting of a finite number of particles $N$, whereas that for the chemical potential has an error of order $N^{-1}$, because in the simulations we change only the volume $V$ of the box leaving the number of particles invariant. (See the Appendix for details). For the equation of state of the pure hard-sphere system we have

$$
z^{l}(\eta, 0)=1+\frac{4 \eta+1.216224 \eta^{2}+1.246720 \eta^{3}}{1-2.195944 \eta+1.210352 \eta^{2}}
$$

valid when the system is a fluid. ${ }^{18}$ It is quadrature to determine the chemical potential

$$
\frac{\mu^{l}(\eta, 0)}{k_{B} T}=\ln \eta-1+z^{l}(\eta, 0)+\int_{0}^{\eta} d \eta^{\prime} \frac{z^{l}\left(\eta^{\prime}, 0\right)-1}{\eta^{\prime}} .
$$

For the pressure of the hard-sphere (fcc) solid we use ${ }^{19}$

$$
z^{s}(\eta, 0)=\frac{3}{1-(6 / \pi \sqrt{2}) \eta}-\frac{0.5921((6 / \pi \sqrt{2}) \eta-0.7072)}{(6 / \pi \sqrt{2}) \eta-0.601},
$$

and for the chemical potential

where $h$ is Planck's constant and $m$ is the mass of a particle. 
TABLE I. Volume fraction of particles in the coexisting fluid and solid phases as a function of $\beta$ and $\kappa$ determined by the simulations. The stickiness parameter is computed via the optimized Baxter model (OBM) and the $\mathrm{B}_{2}$ method $\left(\mathrm{B}_{2}\right)$.

\begin{tabular}{|c|c|c|c|c|c|c|c|c|}
\hline \multirow[b]{2}{*}{$\beta$} & \multicolumn{4}{|c|}{$\kappa=7$} & \multicolumn{4}{|c|}{$\kappa=9$} \\
\hline & $\eta_{l}$ & $\eta_{s}$ & $\tau(\mathrm{OBM})$ & $\tau_{0}\left(\mathrm{~B}_{2}\right)$ & $\eta_{l}$ & $\eta_{s}$ & $\tau(\mathrm{OBM})$ & $\tau_{0}\left(\mathrm{~B}_{2}\right)$ \\
\hline 0 & 0.492 & 0.543 & $\infty$ & $\infty$ & 0.492 & 0.543 & $\infty$ & $\infty$ \\
\hline 0.25 & 0.493 & 0.551 & 8.921 & 1.930 & 0.493 & 0.552 & 6.278 & 2.549 \\
\hline 0.5 & 0.493 & 0.561 & 3.329 & 0.910 & 0.494 & 0.563 & 2.673 & 1.199 \\
\hline 0.75 & 0.492 & 0.571 & 1.689 & 0.570 & 0.494 & 0.576 & 1.521 & 0.750 \\
\hline 1 & 0.490 & 0.584 & 0.986 & 0.400 & 0.492 & 0.591 & 0.970 & 0.526 \\
\hline 1.25 & 0.485 & 0.598 & 0.616 & 0.299 & 0.488 & 0.608 & 0.659 & 0.392 \\
\hline 1.5 & 0.478 & 0.613 & 0.405 & 0.231 & 0.480 & 0.626 & 0.462 & 0.303 \\
\hline 1.75 & 0.465 & 0.627 & 0.271 & 0.184 & 0.464 & 0.643 & 0.328 & 0.240 \\
\hline 2 & 0.441 & 0.641 & 0.183 & 0.148 & 0.437 & 0.657 & 0.236 & 0.193 \\
\hline
\end{tabular}

$$
\begin{aligned}
\frac{\mu^{s}(\eta, 0)}{k_{B} T}= & \ln \eta-1+z^{s}(\eta, 0)+\frac{f^{s}\left(\eta_{0}\right)}{k_{B} T} \\
& +\int_{\eta_{0}}^{\eta} d \eta^{\prime} \frac{z^{s}\left(\eta^{\prime}, 0\right)-1}{\eta^{\prime}}
\end{aligned}
$$

where $f^{s}\left(\eta_{0}\right) / k_{B} T=5.91889(4)$ is the free energy of the hardsphere solid in the thermodynamic limit $N \rightarrow \infty$ at volume fraction $\eta_{0}=0.5450 .{ }^{20}$ We thus calculate the pressure and the chemical potential of the system in the thermodynamic limit using

$$
\frac{P(\eta, \beta) v_{0}}{k_{B} T} \simeq \eta z^{i}(\eta, 0)+\eta^{2} \frac{\partial}{\partial \eta} \int_{0}^{\beta} d \beta^{\prime} \frac{1}{\beta^{\prime}}\left\langle\frac{U_{1}}{k_{B} T}\right\rangle_{N}
$$

and

$$
\frac{\mu(\eta, \beta)}{k_{B} T} \simeq \frac{\mu^{i}(\eta, 0)}{k_{B} T}+\frac{\partial}{\partial \eta} \eta \int_{0}^{\beta} d \beta^{\prime} \frac{1}{\beta^{\prime}}\left\langle\frac{U_{1}}{k_{B} T}\right\rangle_{N},
$$

where $i=s, l$. These expressions are not exact but correct to order $N^{-1}$ because the number of particles in the simulations is finite (see the Appendix for details).

To determine the average energy per particle $\left\langle U_{1} / k_{B} T\right\rangle_{N}$ we need to initiate the simulation by choosing a convenient starting configuration. In the case that the system is a solid, we assume it is a fcc crystal at the appropriate density. For the liquid, a configuration at the required density is initiated by putting the particles in the box at random and then running the simulation until the particles no longer overlap. This is done at a low value of $\beta$ and we then use this starting configuration for all values of $\beta$ at the same density. The simulation is then run for 10000 cycles (i.e., trial moves per particle) at the relevant value of $\beta$ to determine the appropriate maximum displacement of a particle at an acceptance probability of a particle displacement of 0.40 . The maximum displacement is then fixed and the simulation is run for another 10000 cycles for the system to equilibrate. Finally, the average energy per particle is measured every 100 cycles during another 50000 cycles.

To perform the integration in Eq. (18) we run simulations at values of $\beta$ ranging from 0.1 until the appropriate value at intervals of 0.1 . A simulation at $\beta=0.02$ is also performed. We then fit the points to a curve and use this to perform the integration. To determine the density dependence of the free energy $f_{N}$ about a certain density, we compute the free energy at about ten values of the density close to it, at intervals of 0.1 . We again fit these to a curve which is used in Eqs. (25) and (26) to determine the chemical potential and the pressure at the desired density.

\section{RESULTS AND DISCUSSION}

\section{A. Phase equilibrium}

We first test the optimized Baxter model with respect to the fluid phase of hard spheres with Yukawa attraction when it coexists with the solid phase. This coexistence has been studied before via computer simulations, ${ }^{14,15}$ but these papers did not report the pressure and chemical potential, data we do need here.

At a given strength $\beta$ and inverse range $\kappa$ of the attraction, we compute the volume fractions of the coexisting fluid and solid phases by equating the pressures and the chemical potentials in the respective phases. This is done for $\kappa=7$ and 9 and $\beta$ running from 0 to 2 at intervals of 0.25 (see Table I). Our phase boundaries at $\kappa=7$ agree well with those computed earlier by Dijkstra ${ }^{15}$ who used the same method, though with a smaller system of $N=108$ particles. The deviation in volume fraction is at most $2 \%$ (we determined the phase boundaries from a plot presented in Ref. 15, so this may account for part of the difference). At low values of $\beta$, the agreement with the simulations of Hagen and Frenkel ${ }^{14}$ is also good, but with increasing $\beta$ the difference between their phase boundary on the fluid side and ours becomes appreciable until our prediction of the volume fraction is about $20 \%$ higher than theirs at $\beta=2$. We note that in Ref. 14 a different method was used to determine the phase boundary. The phase boundaries on the solid side do agree within $3 \%$. We regain essentially the same picture at $\kappa=9$ though the difference in the phase boundaries at the fluid side is less pronounced (about $14 \%$ at $\beta=2$ ). The phase diagram at $\kappa$ $=9$ was not determined in Ref. 15 .

Next, we use the OBM to determine the effective stickiness parameter $\tau$ [Eq. (11)] and the properties of the fluid at coexistence. By way of comparison, we also evaluate $\tau_{0}$ by 

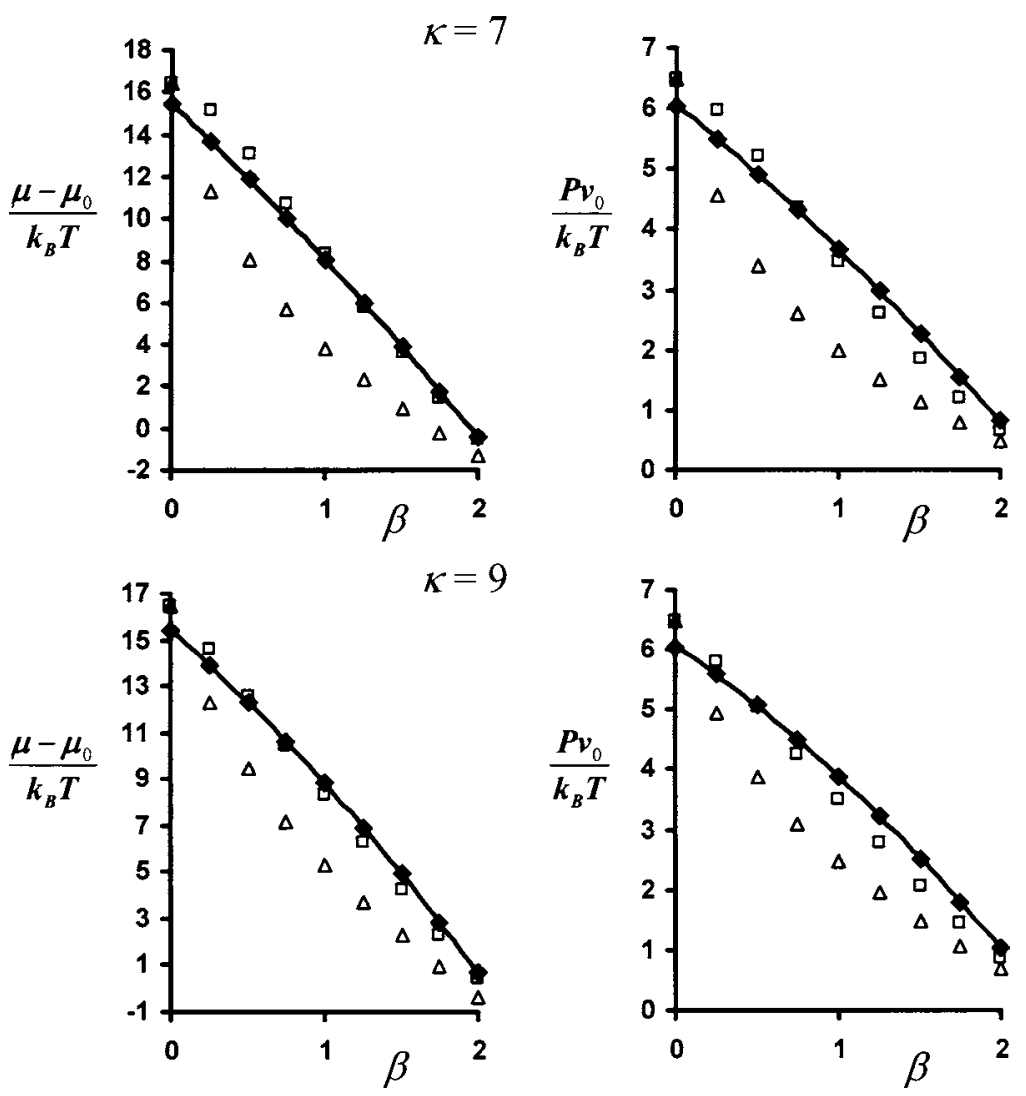

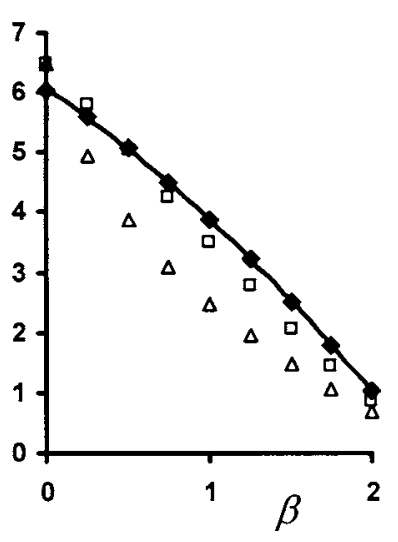

FIG. 1. Dimensionless chemical potential and dimensionless pressure as a function of the strength $\beta$ of the Yukawa potential for the coexisting fluid and solid phases. Here $\kappa=7$ and $\kappa=9$. The diamonds and the fitted line are results from the simulations. The squares are predictions from the optimized Baxter model (at the same densities) and the triangles have been computed by the $B_{2}$ method. equating the respective second virial coefficients of the attractive Yukawa interaction and the Baxter potential [see Eq. (5)] and computing the properties of the resulting Baxter fluid. We will refer to this as the $B_{2}$ method which is strictly correct only at very low concentrations, as we stressed above. We employ Eqs. (13) and (14) to calculate the pressure and the chemical potential from the volume fractions and the respective values of $\tau$ from the two methods. These predictions are compared with the simulations in Fig. 1. It is clear from the figure that the predictions of the OBM are significantly better than those via the $B_{2}$ method along the whole phase boundary. The OBM is actually quite accurate to within a few percent. Recall that at $\beta=0$, i.e., in the absence of attraction, the two volume fractions predicted by the two methods necessarily coincide simply because $\tau=\infty$ in both cases. However, this volume fraction does not agree with that from the simulations which is due to the fact that we use the accurate equation of state [Eq. (21)] in the latter. The analytical theory is, of course, approximate and overestimates the pressure and the chemical potential.

\section{B. Consistency test in the fluid phase}

The Baxter model itself has been solved in the PercusYevick approximation, ${ }^{2}$ and we here use the compressibility route to obtain the thermodynamic properties. We know, however, that in the case of the hard-sphere system, the analytical calculations carried out in this way are too high (e.g., at $\eta=0.4$, both the pressure and the chemical potential are overestimated by $4 \%$ ). We therefore seek to test the argumentation leading to the replacement of the actual fluid by the OBM in a way which is less sensitive to the Percus-
Yevick approximation. For instance, we note that the stickiness parameter $\tau$ in the OBM merely depends on the properties of the distribution function $\widetilde{g}$ very close to the sphere [see Eq. (8)]. Though this does depend on the Percus-Yevick approximation, it stands to reason that the functions $G$ and $H$ are more robust to approximation than the oscillatory behavior which $\tilde{g}$ actually displays in full [and which is implicit in Eqs. (13) and (14)]. Thus, in the following simulations for the fluid phase, we investigate whether mathematical similarity $^{22}$ is achieved with respect to the parameter $\tau$, as given by Eq. (11). This constitutes a reasonable consistency check with regard to the representation of the real fluid by the OBM.

Our procedure is as follows. We start at a given volume fraction. Next we choose a set of values of the inverse range of the Yukawa potential (i.e., $\kappa=5,7,9,11,13$, and 15). We then fix a certain value of the stickiness parameter $\tau$ and compute the concomitant value $\beta$ for each $\kappa$ with the help of Eq. (11). If similarity ${ }^{22}$ does apply, the thermodynamic properties should depend solely on $\tau$ and $\eta$, i.e., they ought to be independent of $\kappa$ at constant $\tau$.

We have performed this test on simulations in a suitable range of volume fractions $\eta$ and stickiness parameters $\tau$ with associated interaction parameters $\kappa$ and $\beta$, as chosen above. (See Figs. 2-4). We have also performed simulations at $\eta$ $=0.1$; the OBM is quantitatively accurate then, as is the $B_{2}$ method (data not shown). In some cases the attraction is so strong in terms of $\beta$ that the simulated fluid is actually in the metastable region with respect to fluid-crystal coexistence. In effect, if the system were macroscopic, phase separation into fluid and crystal phases would occur. We are aware of this on 
$\eta=0.2$
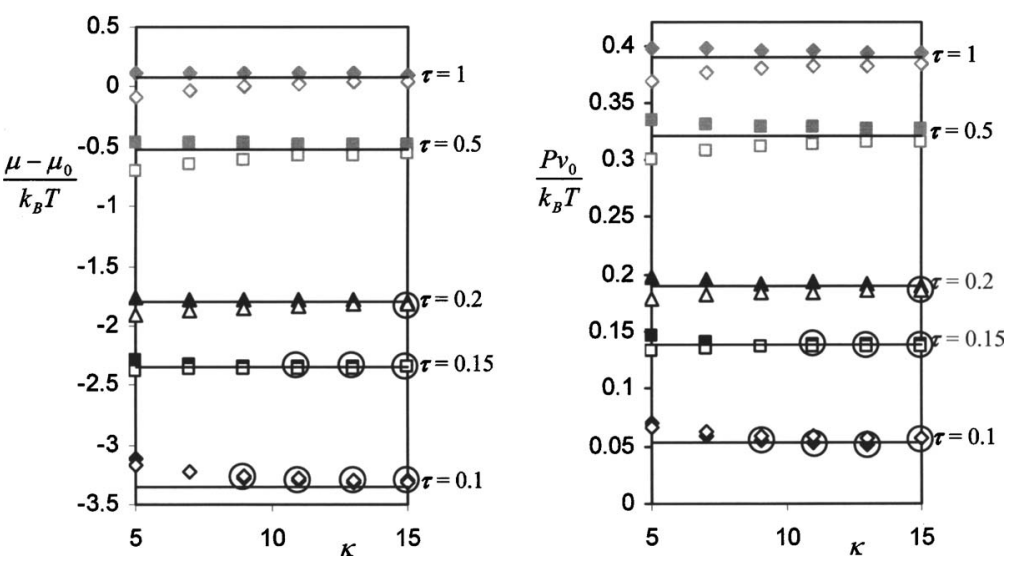

FIG. 2. Dimensionless chemical potential and dimensionless pressure of the fluid phase as a function of the inverse range $\kappa$ of the Yukawa potential at volume fraction $\eta=0.2$. The solid symbols are results from the simulations, the horizontal lines are predictions from the optimized Baxter model at a variety of fixed values of $\tau$. In the simulations the strength of the attraction $\beta$ is chosen in such a way that the optimized Baxter model gives the appropriate value of $\tau$ : gray filled diamonds $\tau=1$, gray filled squares $\tau=0.5$, black filled triangles $\tau=0.2$, black filled squares $\tau=0.15$, and black filled diamonds $\tau=0.1$. The corresponding open symbols have been computed by the $B_{2}$ method. Encircled points are metastable with regard to fluid-crystal coexistence.

the basis of simulations performed by Hagen and Frenkel ${ }^{14}$ and by Dijkstra. ${ }^{15}$ In both these investigations, fluid-crystal coexistence was assessed quantitatively by positing the two phases a priori. We have not done this here because our main interest has been in testing the OBM for the fluid phase. From their data, we judge our simulations to be metastable in this sense for certain points encircled in Figs. 2-4. Despite the preemption of phase separation, we may still determine the pressure and chemical potential as if the phases were stable. The OBM pertains to the fluid phase and cannot address this type of metastability, though invariance of the pressure and chemical potential may be assumed in the two phases.

We first note that the simulated thermodynamic properties are generally quite independent of $\kappa$. (See the filled symbols in Figs. 2-4). This implies that $\tau$ is indeed a useful similarity variable and the OBM is a consistent approximation scheme. The variation in the pressure and chemical potential computed by simulation is only a few percent with few exceptions. Sometimes, there are visible deviations from the horizontal at low values of $\kappa$, for instance, when the volume fraction is 0.4 . By contrast, there are marked devia- tions from similarity when the attraction is strong $(\tau=0.1)$ at a volume fraction of $\eta=0.4$ at $\kappa=11$ and 13 (see Fig. 4). Mere visual scrutiny of the simulation snapshots shows that gelation seems to be occurring-note that the attraction is so strong that we are now well beyond the percolation threshold. $^{12}$ This refers to a second type of metastability. It is beyond the scope of this paper to investigate this phenomenon further or the possibility of fluid-fluid coexistence.

Next, it is of interest to compare the magnitudes of the simulated thermodynamic properties with those computed with the help of the OBM (see the curves in Figs. 2-4 which are horizontal because $\tau$ was forced to be constant in each case). The analytical predictions are virtually quantitative, except at those densities at $\tau=0.1$ where gelation seems to occur, as discussed above and with regard to some of the pressures at higher concentrations. The latter are overestimated at $\tau=0.5$ and 1 in Figs. 3 and 4 which we attribute to deficiencies in the Baxter model itself (i.e., the PercusYevick approximation), since the simulational data are quite independent of $\kappa$, as stressed above.

For the sake of comparison we have also displayed thermodynamic properties computed by the $B_{2}$ method. At a cer-

$$
\eta=0.3
$$
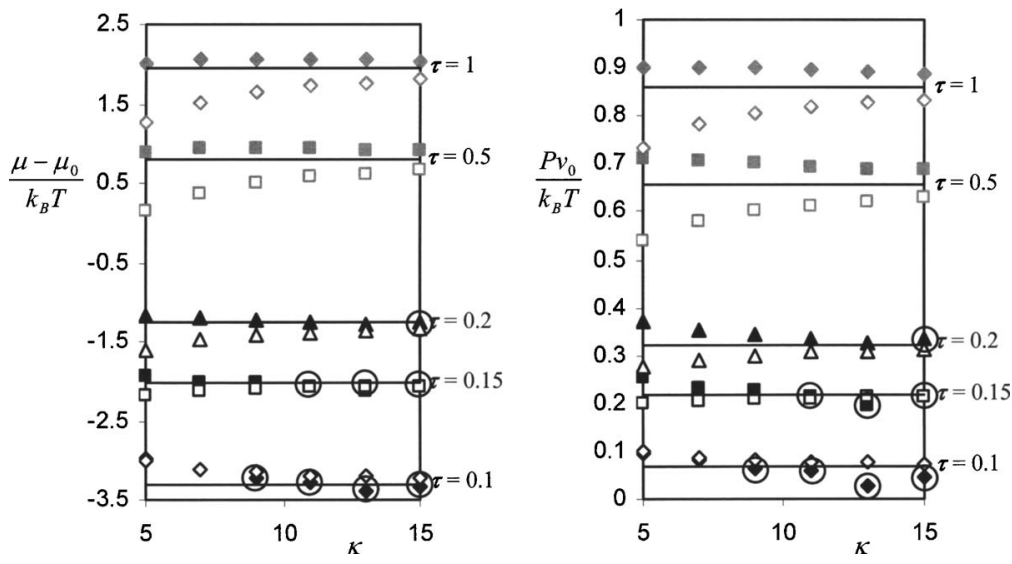

FIG. 3. Same as Fig. 2 but now at volume fraction $\eta=0.3$. 


\section{$\eta=0.4$}
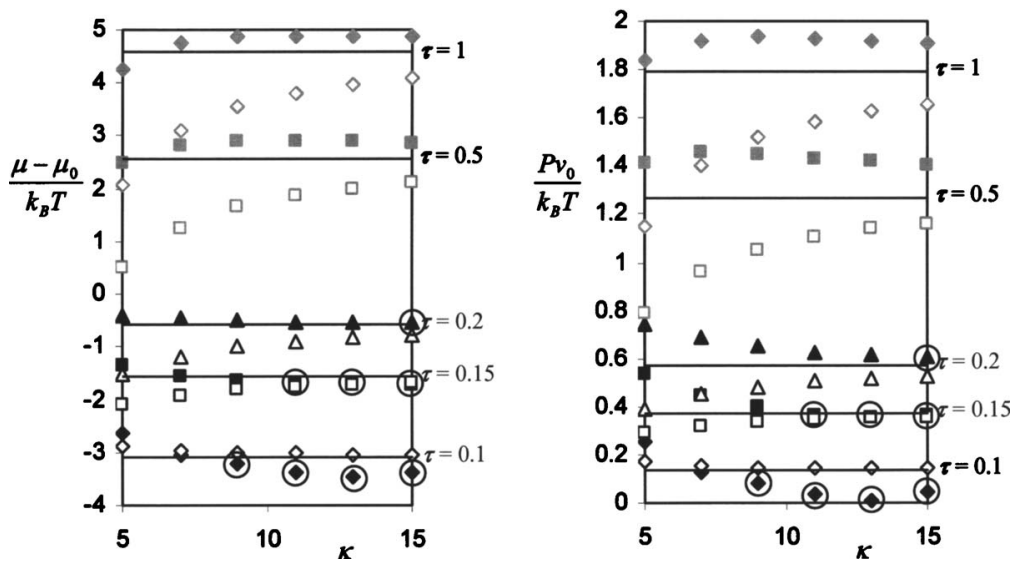

FIG. 4. Same as Fig. 2 but now at volume fraction $\eta=0.4$. tain $\kappa$ and $\beta$ we evaluate $\tau_{0}$ with the help of Eq. (5) using the Yukawa interaction [Eq. (1)] (thus $\tau_{0}$ is not constant like $\tau$ ) and then calculate the pressure and chemical potential within the Baxter model. The $B_{2}$ method works well at $\eta=0.1$ (data not shown), which is not surprising since neglecting to variationally adjust virials higher than second is not so crucial in this case. However, the $B_{2}$ method worsens progressively as the concentration increases and ultimately becomes unreliable (see Figs. 2-4). This is of course expected: the $B_{2}$ method merely adjusts a single coefficient $B_{2}$, whereas the free energy itself is variationally optimized in the OBM.

We conclude that the optimized Baxter model is a convenient quantitative, analytical theory for computing the thermodynamic properties of a fluid of hard spheres interacting by an attraction of short range. Moreover, the variational scheme used in deriving the OBM is consistent, especially when the range of the potential is short, i.e., less than approximately $10 \%$ of the particle diameter $(\kappa \gtrsim 10)$. Overall, the OBM is accurate to within $10 \%$, except under some conditions of very strong attraction at high volume fractions $(\tau=0.1, \eta=0.3$ and 0.4$)$, and it is actually much more precise in most cases.

\section{ACKNOWLEDGMENTS}

The work of the FOM Institute is part of the research program of the Foundation for Fundamental Research on Matter (FOM) and was made possible through financial support by the Dutch Foundation for Scientific Research (NWO)

\section{APPENDIX A: FINITE SIZE EFFECTS}

Here we show that the error incurred in Eq. (20) for the chemical potential of the $N$-particle system is of order $N^{-1}$, whereas Eq. (19) for the equation of state is exact. We also prove that the error in the free energy of the system is of order $N^{-1}$.

Our simulations are carried out at a constant number of particles $N$. Hence, we modify the volume fraction $\eta$ by altering the volume of the simulation box. The free energy difference per particle,

$$
\begin{aligned}
\Delta f_{N}(\eta, \beta) & \equiv f_{N}(\eta, \beta)-f_{N}(\eta, 0) \\
& =k_{B} T \int_{0}^{\beta} d \beta^{\prime} \frac{1}{\beta^{\prime}}\left\langle\frac{U_{1}}{k_{B} T}\right\rangle_{N},
\end{aligned}
$$

is determined as a function of the volume fraction, so in effect it is a function of $\eta$ (or $\rho$ ) and $N$ (and of course $\beta$ and $\kappa)$. The exact equation of state $z_{N}(\eta, \beta)$ for the $N$-particle system is then

$$
\begin{aligned}
z_{N}(\eta, \beta) & \equiv-\frac{1}{\rho k_{B} T}\left(\frac{\partial F_{N}(\eta, \beta)}{\partial V}\right)_{N, T} \\
& =z_{N}(\eta, 0)+\eta \frac{\partial}{\partial \eta} \frac{\Delta f_{N}(\eta, \beta)}{k_{B} T},
\end{aligned}
$$

where $F_{N}(\eta, \beta)=N f_{N}(\eta, \beta)$ and the exact chemical potential is

$$
\begin{aligned}
\frac{\mu_{N}(\eta, \beta)}{k_{B} T} \equiv & \frac{1}{k_{B} T}\left(\frac{\partial F_{N}(\eta, \beta)}{\partial N}\right)_{V, T} \\
= & \frac{\mu_{N}(\eta, 0)}{k_{B} T}+\frac{\partial}{\partial \eta} \frac{\eta \Delta f_{N}(\eta, \beta)}{k_{B} T} \\
& -\frac{1}{N} \frac{\partial}{\partial N^{-1}} \frac{\Delta f_{N}(\eta, \beta)}{k_{B} T} .
\end{aligned}
$$

Here, and in the rest of the Appendix, we have switched to

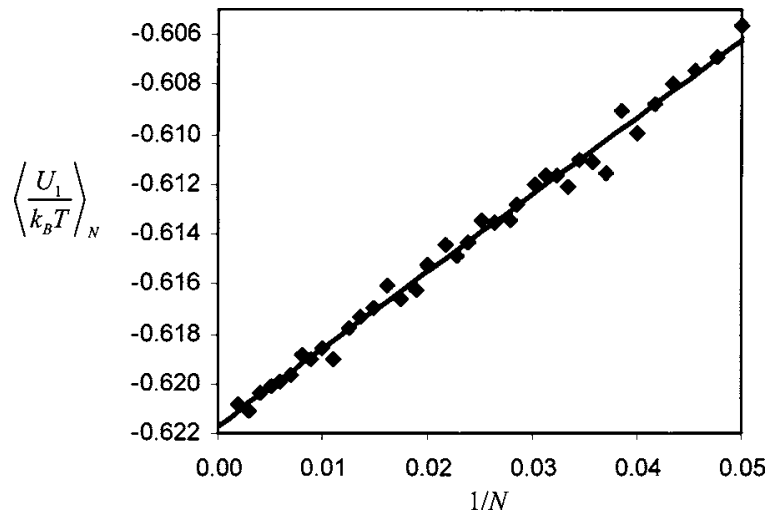

FIG. 5. Example of the dependence of the average dimensionless energy per particle $\left\langle U_{1} / k_{B} T\right\rangle_{N}$ in the fluid on the size of the system. Here $\kappa=9, \beta=1$, and $\eta=\pi / 15 \approx 0.20944\left[\rho(2 a)^{3}=0.4\right] . N$ denotes the number of particles. 


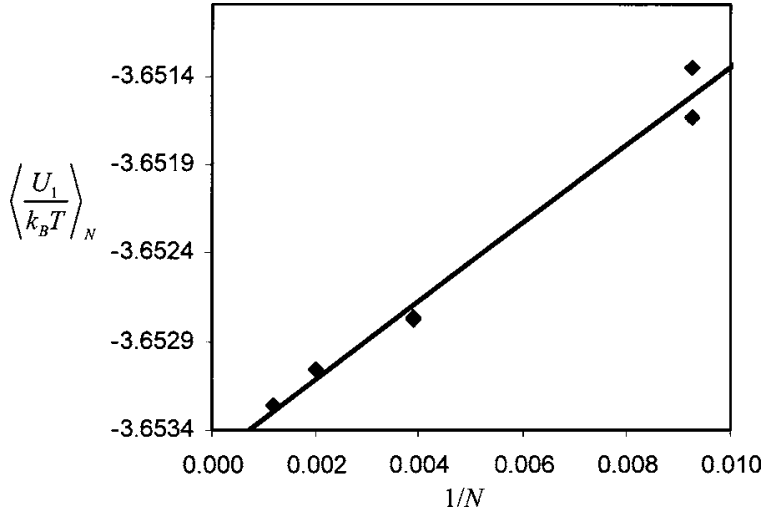

FIG. 6. Same as Fig. 5 but now for the solid at $\eta=\pi / 5 \approx 0.62832$ $\left[\rho(2 a)^{3}=1.2\right]$.

the new independent variables $\eta$ and $N$ so that derivatives with respect to $\eta$ are taken at constant $N$ and derivatives with respect to $N$ are taken at constant $\eta$. We see from Eqs. (A2) and (A3) that Eq. (20) has an error of order $N^{-1}$, whereas Eq. (19) is exact.

We now assume that we may Taylor expand $\Delta f_{N}(\eta, \beta)$ for small values of $N^{-1}$ at constant volume fraction. It is not obvious that this is allowed. In the case of a crystal, for example, the first-order correction to the free energy per particle due to the fact that the number of particles is finite is of order $N^{-1} \ln N .^{20,21}$ This correction is the same for systems of identical numbers of particles, however, regardless of the interaction. Since our $f_{N}$ is the difference in the free energies per particle pertaining to the two respective crystals (with different pair potentials), the $O\left(N^{-1} \ln N\right)$ corrections simply cancel. Moreover, from Ref. 20 we know that the leading higher order corrections to the free energy per particles are of order $N^{-1}$. These deliberations are confirmed in Figs. 5 and 6, which show that the leading corrections to the average dimensionless energy per particle $\left\langle U_{1} / k_{B} T\right\rangle_{N}$ are indeed of or$\operatorname{der} N^{-1}$ at the representative values $\beta=1, \kappa=15$, and $\rho(2 a)^{3}=0.4(\eta=\pi / 15 \approx 0.20944)$ for the fluid and $\rho(2 a)^{3}$ $=1.2(\eta=\pi / 5 \approx 0.62832)$ for the solid. Therefore, we conclude that the free energy per particle in a system containing an infinite number of particles is given by

$$
\Delta f_{\infty}(\eta, \beta)=\Delta f_{N}(\eta, \beta)+O\left(\frac{1}{N}\right) .
$$

In the same manner, the equation of state $z_{\infty}(\eta, \beta)$ is then

$$
z_{\infty}(\eta, \beta)=z_{\infty}(\eta, 0)+\eta \frac{\partial}{\partial \eta} \frac{\Delta f_{N}(\eta, \beta)}{k_{B} T}+O\left(\frac{1}{N}\right),
$$

and the chemical potential is expressed by

$$
\frac{\mu_{\infty}(\eta, \beta)}{k_{B} T}=\frac{\mu_{\infty}(\eta, 0)}{k_{B} T}+\frac{\partial}{\partial \eta} \frac{\eta \Delta f_{N}(\eta, \beta)}{k_{B} T}+O\left(\frac{1}{N}\right) .
$$

\section{APPENDIX B: SECOND-ORDER CORRECTION TO THE FREE ENERGY}

We estimate the second-order correction to the free energy (see Appendix C of Ref. 1)

$$
\Delta=\frac{9}{4} \eta^{2} Y \text {. }
$$

This correction leads for instance to a correction to the dimensionless pressure $P v_{0} / k_{B} T$ approximately equal to $-2 \eta \Delta .{ }^{1}$ The first part of the analysis in Appendix $C$ of Ref. 1 is also useful here and we again approximate $Y$ by

$$
\begin{aligned}
Y \simeq & \frac{2}{3}\left(9 G+10 G H-12+\frac{\lambda}{2}\right)\left[\int_{2}^{\infty} d t t B(t)\right]^{2} \\
+ & {\left[\int_{2}^{\infty} d t t B(t)\right]\left[\int_{2}^{\infty} d s s^{3} B(s)\right], }
\end{aligned}
$$

where

$$
B(x) \equiv g(x)\left[\exp \left(-\frac{U_{Y}(x)}{k_{B} T}+\frac{U_{\mathrm{AHS}}(x)}{k_{B} T}\right)-1\right]
$$

and $\lambda, G$, and $H$ are given by Eqs. (7)-(10). We split the pair distribution function $g(x)$ in the reference state into $g_{\omega}(x)$ and a regular part $\widetilde{g}(x)$ given by Eq. (8) (see also Ref. 1):

$$
g(x)=\tilde{g}(x)+g_{\omega}(x),
$$

with

$$
g_{\omega}(x)= \begin{cases}0, & x<2 \\ \frac{\lambda(2+\omega)}{12 \omega}+O(1), & 2 \leqslant x \leqslant 2+\omega \\ 0, & x>2+\omega .\end{cases}
$$

We then insert the expressions for the potentials Eqs. (1) and (3) into Eq. (B3) and derive in the limit $\omega \rightarrow 0$

TABLE II. Estimates of the second-order correction $\Delta$ to the free energy for $\eta=0.4$ at various $\tau$ and $\kappa . \Delta$ is determined from Eq. (B15) within the approximation given by Eq. (B14)

\begin{tabular}{cccccccc}
\hline \hline$\tau$ & $\kappa$ & 5 & 7 & 9 & 11 & 13 & 15 \\
\hline \multirow{2}{*}{0.1} & $\beta$ & 1.966 & 2.447 & 2.805 & 3.088 & 3.321 & 3.519 \\
& $\Delta$ & -0.1315 & -0.0637 & -0.0352 & -0.0214 & -0.0139 & -0.0096 \\
0.15 & $\beta$ & 1.725 & 2.099 & 2.397 & 2.644 & 2.855 & 3.038 \\
& $\Delta$ & -0.2129 & -0.0944 & -0.0505 & -0.0303 & -0.0197 & -0.0135 \\
0.2 & $\beta$ & 1.548 & 1.853 & 2.110 & 2.332 & 2.526 & 2.697 \\
& $\Delta$ & -0.2762 & -0.1154 & -0.0603 & -0.0360 & -0.0233 & -0.0160 \\
0.5 & $\beta$ & 1.017 & 1.140 & 1.282 & 1.423 & 1.558 & 1.686 \\
& $\Delta$ & -0.4291 & -0.1467 & -0.0715 & -0.0416 & -0.0268 & -0.0186 \\
1 & $\beta$ & 0.694 & 0.727 & 0.803 & 0.891 & 0.981 & 1.070 \\
& $\Delta$ & -0.4209 & -0.1211 & -0.0549 & -0.0312 & -0.0200 & -0.0139 \\
\hline \hline
\end{tabular}




$$
\begin{aligned}
\int_{2}^{\infty} d x x B(x)= & -\frac{\lambda}{3}+G \int_{2}^{\infty} d x x(1+H(x-2)) \\
& \times\left(e^{-U_{Y}(x) / k_{B} T}-1\right)+O\left(e^{-\kappa}\right)
\end{aligned}
$$

and

$$
\begin{aligned}
\int_{2}^{\infty} d x x^{3} B(x)= & -\frac{4 \lambda}{3}+G \int_{2}^{\infty} d x x^{3}(1+H(x-2)) \\
& \times\left(e^{-U_{Y}(x) / k_{B} T}-1\right)+O\left(e^{-\kappa}\right)
\end{aligned}
$$

In both cases the integration on the right hand side should run from $x=2$ to $x=4$, so extending the integrals to $\infty$ only introduces errors of order $e^{-\kappa}$. In the OBM, $\tau$ is determined by the condition that the first-order correction to the free energy vanishes

$$
\begin{aligned}
\int_{2}^{\infty} d x x^{2} B(x)= & -\frac{2 \lambda}{3}+G \int_{2}^{\infty} d x x^{2}(1+H(x-2)) \\
& \times\left(e^{-U_{Y}(x) / k_{B} T}-1\right)+O\left(e^{-\kappa}\right)=0 .
\end{aligned}
$$

This expression is used to rewrite Eqs. (B6) and (B7)

$$
\begin{aligned}
\int_{2}^{\infty} d x x B(x)= & -\frac{1}{2} G \int_{2}^{\infty} d x x(x-2)(1+H(x-2)) \\
& \times\left(e^{-U_{Y}(x) / k_{B} T}-1\right)+O\left(e^{-\kappa}\right) \\
\int_{2}^{\infty} d x x^{3} B(x)= & G \int_{2}^{\infty} d x x^{2}(x-2)(1+H(x-2)) \\
& \times\left(e^{-U_{Y}(x) / k_{B} T}-1\right)+O\left(e^{-\kappa}\right)
\end{aligned}
$$

which are readily approximated. We substitute $y=\exp [$ $-\kappa(x / 2-1)]$ which ultimately leads to

$$
\int_{2}^{\infty} d x x B(x)=\frac{4 G}{\kappa^{2}} J_{1}(\beta)+O\left(\kappa^{-3}\right)
$$

and

$$
\int_{2}^{\infty} d x x^{3} B(x)=-\frac{16 G}{\kappa^{2}} J_{1}(\beta)+O\left(\kappa^{-3}\right) .
$$

Here we have introduced

$$
J_{1}(\beta) \equiv-\int_{0}^{1} d y \frac{e^{\beta y}-1}{y} \ln y
$$

An approximation for $J_{1}(\beta)$ that is accurate to within $1.4 \%$ in the relevant range of $0 \leqslant \beta \leqslant 3.52$ is given by

$$
J_{1}(\beta) \simeq \begin{cases}\beta+\frac{1}{8} \beta^{2}, & 0 \leqslant \beta<0.8 \\ 2.81\left(e^{0.34 \beta}-1\right), & 0.8 \leqslant \beta \leqslant 3.52 .\end{cases}
$$

Finally, we insert Eqs. (B11) and (B12) into Eq. (B2). We thus obtain an approximation for the second-order correction to the free energy

$$
\Delta \simeq \frac{24 G^{2}}{\kappa^{4}}\left(9 G+10 G H-18+\frac{\lambda}{2}\right) J_{1}^{2}(\beta) \eta^{2} .
$$

In Table II we present typical values of $\Delta$. The corrections to the pressure are very small (compare with Fig. 1).

${ }^{1}$ P. Prinsen and T. Odijk, J. Chem. Phys. 121, 6525 (2004).

${ }^{2}$ R. J. Baxter, J. Chem. Phys. 49, 2770 (1968).

${ }^{3}$ R. O. Watts, D. Henderson, and R. J. Baxter, Adv. Chem. Phys. 21, 421 (1971).

${ }^{4}$ B. Barboy, J. Chem. Phys. 61, 3194 (1974).

${ }^{5}$ P. T. Cummings, J. W. Perram, and E. R. Smith, Mol. Phys. 31, 535 (1976).

${ }^{6}$ B. Barboy and R. Tenne, Chem. Phys. 38, 369 (1979).

${ }^{7}$ A. J. Post and E. D. Glandt, J. Chem. Phys. 84, 4585 (1986).

${ }^{8}$ G. Stell, J. Stat. Phys. 63, 1203 (1991).

${ }^{9}$ D. Gazzillo and A. Giacometti, J. Chem. Phys. 120, 4742 (2004).

${ }^{10}$ N. A. Seaton and E. D. Glandt, J. Chem. Phys. 87, 1785 (1987).

${ }^{11}$ W. G. T. Kranendonk and D. Frenkel, Mol. Phys. 64, 403 (1988).

${ }^{12}$ M. A. Miller and D. Frenkel, J. Chem. Phys. 121, 535 (2004).

${ }^{13}$ M. A. Miller and D. Frenkel, J. Phys.: Condens. Matter 16, S4901 (2004).

${ }^{14}$ M. H. J. Hagen and D. Frenkel, J. Chem. Phys. 101, 4093 (1994).

${ }^{15}$ M. Dijkstra, Phys. Rev. E 66, 021402 (2002).

${ }^{16}$ H. C. Andersen, J. D. Weeks, and D. Chandler, Phys. Rev. A 4, 1597 (1971).

${ }^{17}$ D. Frenkel and B. Smit, Understanding Molecular Simulation (Academic, San Diego, 2002).

${ }^{18}$ R. J. Speedy, J. Phys.: Condens. Matter 9, 8591 (1997).

${ }^{19}$ R. J. Speedy, J. Phys.: Condens. Matter 10, 4387 (1998).

${ }^{20}$ J. M. Polson, E. Trizac, S. Pronk, and D. Frenkel, J. Chem. Phys. 112, 5339 (2000).

${ }^{21}$ W. G. Hoover, J. Chem. Phys. 49, 1981 (1968).

${ }^{22}$ The concept of "mathematical similarity" or "similarity" is well explained in G. I. Barenblatt, Similarity, Self-similarity and Intermediate Asymptotics (Consultant Bureau, N.Y., 1979). For instance, in our case, the pressure which was originally some function of $\eta, \beta$, and $\kappa$, is proposed to be a function of $\eta$ and $\tau$ only, via argumentation based on the optimized Baxter model. Hence, a system defined by $\eta_{1}, \beta_{1}$, and $\kappa_{1}$ is mathematically similar to that defined by $\eta_{1}, \beta_{2}$, and $\kappa_{2}$ if $\tau\left(\beta_{1}, \kappa_{1}\right)$ $=\tau\left(\beta_{2}, \kappa_{2}\right)$. 